\title{
NEW PARAMETERS OF TEICHMÜLLER SPACES OF RIEMANN SURFACES OF GENUS TWO
}

\author{
Chen Min \\ Peking University, Department of Mathematics \\ Beijing 100871, China
}

\begin{abstract}
In this note, new parameters for the Teichmüller space of a compact Riemann surface of genus 2 are presented. The parameter space is $\mathbf{R}_{+}^{6}$. Given any point in $\mathbf{R}_{+}^{6}$, we have a concrete way of deciding the generators for the corresponding marked Fuchsian group.
\end{abstract}

\section{Introduction}

One important topic in the study of Teichmüller spaces is the parametrization problem. The idea behind it is to shift the focus of research from complicated objects onto easier ones. To be precise, let $T_{g}$ be the Teichmüller space of a compact Riemann surface of genus $g$. We want to find out a parameter space $P$ and a correspondence between $P$ and $T_{g}$. Naturally, a successful parametrization should satisfy:

(1) $P$ is simple and $\operatorname{dim} P=\operatorname{dim} T_{g}$;

(2) the correspondence is not difficult to handle.

Up to now, there have been several well-known parametrizations with important applications. However, these parametrizations are mainly of theoretic importance, and it is difficult to use them for some other purposes like numerical calculations. Recently, B. Maskit gave new parametrizations for Teichmüller spaces of Riemann surfaces which have signatures $(0,4)$ and $(1,1)$. His parametrizations have the following features:

(1) $P=$ half space, $\operatorname{dim} P$ being equal to the dimension of the corresponding Teichmüller space;

(2) given any point in $P$, there is a concrete way of describing the generators in the corresponding marked group.

These characteristics provide a clearer model for the considered Teichmüller spaces and also a practical way of doing numerical calculation.

In this note, we present a parametrization for $T_{2}$, the Teichmüller space of a compact Riemann surface of genus 2. Our parameter space is $\mathbf{R}_{+}^{6}$, and given any point in $\mathbf{R}_{+}^{6}$, we have a concrete way of describing the generators for the

1991 Mathematics Subject Classification: Primary 30C65; Secondary 57N50.

Supported by the Väisälä Foundation and the Finnish Society of Sciences and Letters.

doi:10.5186/aasfm.1992.1718 
corresponding marked Fuchsian group. So far the main method for constructing Fuchsian groups has been combination theorems. The method used in this note yields a different way of obtaining all Fuchsian groups which can be geometrically realized by compact Riemann surfaces of genus 2 .

Having finished the first draft of this note, the author heard in Montpellier in September 1991 that B. Maskit has also recently studied the same problem by using a different approach. The author thanks B. Maskit for encouragement.

\section{Preliminaries}

Let $H$ denote the upper-half plane equipped with the hyperbolic metric. Let $\mathscr{M}$ be the set of all hyperbolic Möbius transformations fixing $H$. Any $g \in \mathscr{M}$ can be written in the form

$$
g(z)=\frac{a z+b}{c z+d}, \quad a d-b c \neq 0,
$$

where $a, b, c$ and $d$ are real numbers. We can associate $g$ with a matrix

$$
\tilde{g}=\left(\begin{array}{ll}
a & b \\
c & d
\end{array}\right)
$$

in $G L(2: R)$. This is not unique. Assume that $g$ is an element in $\mathscr{M}$. Denote by $a(g), r(g)$ the attracting and repelling fixed points of $g$. The multiplier $k(g)$ is given by the cross-ratio

$$
k(g)=(g(z), z, r(g), a(g))=\frac{g(z)-r(g)}{g(z)-a(g)} \frac{z-a(g)}{z-r(g)},
$$

where $z$ is any point in $\hat{\mathbf{C}}$ not fixed by $g$. Especially, $k(g)>1$. The transformation $g$ is completely determined by $a(g), r(g)$ and $k(g)$. In fact,

$$
g(z)=\frac{(k(g) a(g)-r(g)) z-a(g) r(g)(k(g)-1)}{(k(g)-1) z+a(g)-k(g) r(g)} .
$$

The axis of $g, a x(g)$, is the unique geodesic on $H$ with $a(g)$ and $r(g)$ as its terminal points. Denote the commutator $g_{1} \circ g_{2}^{-1} \circ g_{1}^{-1} \circ g_{2}$ of two Möbius transformations $g_{1}$ and $g_{2}$ by $\left[g_{1}, g_{2}\right]$.

Let $R=H / G$ be a compact Riemann surface of genus 2 , where $G$ is a Fuchsian group acting on $H$. It is well-known that $G$ has only hyperbolic elements and that there exist four generators $h_{1}^{0}, g_{1}^{0}, h_{2}^{0}$, and $g_{2}^{0}$ such that $\left[h_{1}^{0}, g_{1}^{0}\right] \circ\left[h_{2}^{0}, g_{2}^{0}\right]=$ id and the points below appear on the boundary of $H$ in clockwise order: 
$r\left(\left[h_{2}^{0}, g_{2}^{0}\right]\right), a\left(h_{1}^{0}\right), a\left(g_{1}^{0}\right), r\left(h_{1}^{0}\right), r\left(g_{1}^{0}\right), a\left(\left[h_{2}^{0}, g_{2}^{0}\right]\right), a\left(h_{2}^{0}\right), a\left(g_{2}^{0}\right), r\left(h_{2}^{0}\right)$, $r\left(g_{2}^{0}\right)$. We can normalize $\left\{h_{j}^{0}, g_{j}^{0}\right\}$ so that $a\left(\left[h_{2}^{0}, g_{2}^{0}\right]\right)=0, r\left(\left[h_{2}^{0}, g_{2}^{0}\right]\right)=\infty$, and $a\left(h_{2}^{0}\right)=1$. See Figure 1 below:

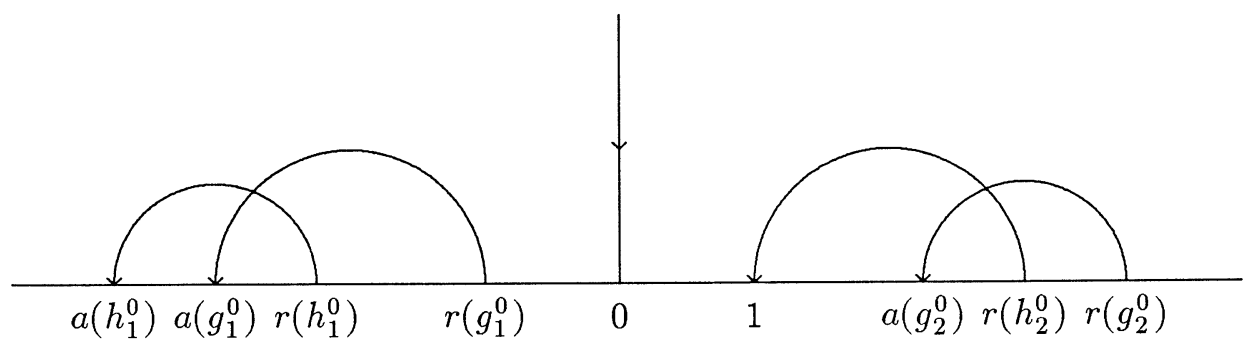

Figure 1.

Denote by $Q$ the set of all quasiconformal mappings which fix $0,1, \infty$ and $H$. Let $f_{1}, f_{2} \in Q ;$ throughout this paper $f_{1} \circ\left(h_{j}, g_{j}\right) \circ f_{1}^{-1}=f_{2} \circ\left(h_{j}, g_{j}\right) \circ f_{2}^{-1}$ will mean

$$
f_{1} \circ h_{j} \circ f_{1}^{-1}=f_{2} \circ h_{j} \circ f_{2}^{-1}, \quad f_{1} \circ g_{j} \circ f_{1}^{-1}=f_{2} \circ g_{j} \circ f_{2}^{-1}, \quad j=1,2 .
$$

The Teichmüller space of $R$ is defined as follows:

$$
T_{2}=\left\{\left(h_{j}, g_{j}\right):\left(h_{j}, g_{j}\right)=f \circ\left(h_{j}^{0}, g_{j}^{0}\right) \circ f^{-1}, f \in Q\right\} .
$$

Note that if $\left(h_{j}, g_{j}\right) \in T_{2}$, then $a x\left(h_{j}\right), a x\left(g_{j}\right)$, and $a x\left(\left[h_{2}, g_{2}\right]\right)$ will form a figure on $H$ similar to Figure 1.

\section{Parametrization of $T_{2}$}

Lemma 1. Let $h, g$, and $P$ in $\mathscr{M}$ satisfy:

(1) $a x(h)$ intersects $a x(g)$,

(2) $h \circ g^{-1} \circ h^{-1} \circ g=P$,

(3) $P(z)=\lambda^{-2} z, \lambda>0$.

Then

$$
k(h)=\frac{a(h)-\lambda r(h)}{r(h)-\lambda a(h)}, \quad k(g)=\frac{a(g)-\lambda r(g)}{r(g)-\lambda a(g)} .
$$

Proof. By Lemma 3.4 in [7], we have

$$
\tilde{h} \tilde{g}^{-1} \tilde{h}^{-1} \tilde{g}=\left(\begin{array}{cc}
-\lambda^{-1} & 0 \\
0 & -\lambda
\end{array}\right),
$$

where

$$
\begin{gathered}
\tilde{h}^{-1}=\left(\begin{array}{cc}
k(h) r(h)-a(h) & -a(h) r(h)(k(h)-1) \\
k(h)-1 & r(h)-k(h) a(h)
\end{array}\right), \\
\tilde{g}^{-1}=\left(\begin{array}{cc}
k(g) r(g)-a(g) & -a(g) r(g)(k(g)-1) \\
k(g)-1 & r(g)-k(g) a(g)
\end{array}\right) .
\end{gathered}
$$


The conclusion follows from the trace equalities

$$
\operatorname{tr}(\tilde{h})=\operatorname{tr}\left[\tilde{h}^{-1}\left(\begin{array}{cc}
-\lambda^{-1} & 0 \\
0 & -\lambda
\end{array}\right)\right] \text { and } \operatorname{tr}(\tilde{g})=\operatorname{tr}\left[\left(\begin{array}{cc}
-\lambda^{-1} & 0 \\
0 & -\lambda
\end{array}\right) \tilde{g}^{-1}\right]
$$

respectively. Q.E.D.

Denote by $\mathscr{D}$ the set of all pairs $(h, g)$ in $\mathscr{M} \times \mathscr{M}$ with the property

$$
a(h)<a(g)<r(h)<r(g) .
$$

Lemma 2. Let $P(z)=\lambda^{-2} z, \lambda>1, z \in \mathbf{C}$. Given three points $a, p$ and $r$ on the real axis satisfying

$$
0<a<p<r<\lambda a,
$$

there exists a unique pair $(h, g) \in \mathscr{D}$ such that

$$
h \circ g^{-1} \circ h^{-1} \circ g=P, \quad \text { and } \quad a(h)=a, \quad r(h)=r, \quad a(g)=p .
$$

Conversely, if $(h, g) \in \mathscr{D}$ satisfies $h \circ g^{-1} \circ h^{-1} \circ g=P, 0<a(h)$, then

$$
0<a(h)<a(g)<r(h)<\lambda a(h) .
$$

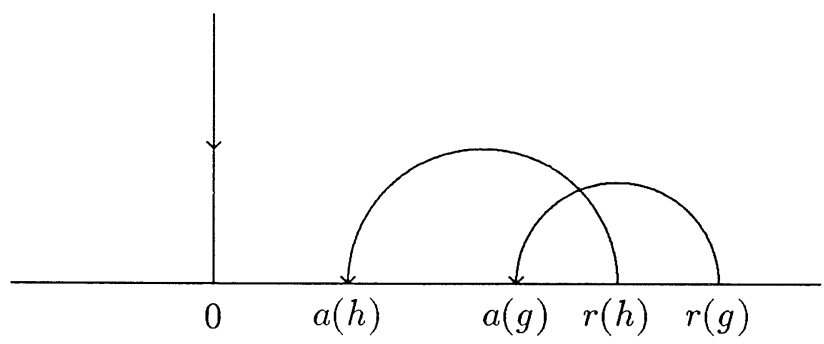

Figure 2.

Proof. The set $S=\{(a, p, r): 0<a<p<r<\lambda a\}$ is connected and open in $\mathbf{R}^{3}$. Clearly there are $\left(a_{0}, p_{0}, r_{0}\right) \in S$ and $\left(h_{0}, g_{0}\right) \in \mathscr{D}$ such that (3.2) holds. Assume that $(a, p, r)$ is sufficiently close to $\left(a_{0}, p_{0}, r_{0}\right)$. We get $h$ as follows:

$$
a(h)=a, \quad r(h)=r, \quad k(h)=\frac{a-\lambda r}{r-\lambda a} .
$$

By the same kind of computation as in Lemma 1 we get

$$
k(h)=k\left(h^{-1} \circ P\right) .
$$


Since $(a, p, r)$ is close to $\left(a_{0}, p_{0}, r_{0}\right)$ and the attracting and repelling fixed points of $h^{-1} \circ P$ are close to the attracting and repelling fixed points of $h_{0}^{-1} \circ P$, respectively, we see that there exists a unique $g \in \mathscr{M}$, which is not identity, such that

$$
g^{-1}(a)=r\left(h^{-1} \circ P\right), \quad g^{-1}(r)=a\left(h^{-1} \circ P\right), \quad g^{-1}(p)=p .
$$

This together with (3.3) implies that $g^{-1} \circ h^{-1} \circ g=h^{-1} \circ P$. Clearly the assumption that $(a, p, r)$ is close to $\left(a_{0}, p_{0}, r_{0}\right)$ implies that $(a(h), r(h), a(g), r(g))$ is close to $\left(a\left(h_{0}\right), r\left(h_{0}\right), a\left(g_{0}\right), r\left(g_{0}\right)\right)$, which yields $(h, g) \in \mathscr{D}$. Let $S_{1}$ be the subset of $S$ whose elements can be realized by (3.2). From the previous discussion we know that $S_{1}$ is a non-empty open set in $S$. Now we assume that there exist $(a, p, r) \in S$ and $\left(a_{n}, p_{n}, r_{n}\right) \in S_{1}(n=1,2, \ldots)$ such that $\left(a_{n}, p_{n}, r_{n}\right) \rightarrow(a, p, r)$, $n \rightarrow \infty$. Consider the corresponding pairs $\left(h_{n}, g_{n}\right) \in \mathscr{D}, n=1,2, \ldots$ They satisfy

$$
\begin{gathered}
g_{n}^{-1} \circ h_{n}^{-1} \circ g_{n}=h_{n}^{-1} \circ P, \\
0<a\left(h_{n}\right)=a_{n}<r_{n}=r\left(h_{n}\right)<\lambda a_{n}, \\
a_{n}<p_{n}=a\left(g_{n}\right)<r_{n}<r\left(g_{n}\right)<\lambda p_{n},
\end{gathered}
$$

where the last inequality follows from Lemma 1 and the fact $k\left(g_{n}\right)>1$. We may assume that $r\left(g_{n}\right) \rightarrow \tilde{r} \in[r, \lambda p]$, and $k\left(g_{n}\right) \rightarrow k \in[1,+\infty]$. It follows that

$$
\lim _{n \rightarrow \infty} k\left(g_{n}\right)=\varliminf_{n \rightarrow \infty} \frac{p_{n}-\lambda r\left(g_{n}\right)}{r\left(g_{n}\right)-\lambda p_{n}} \geq \frac{p-\lambda r}{r-\lambda p}>1,
$$

and $k=+\infty$ if and only if $\tilde{r}=\lambda p$. If $\tilde{r}=r$, we have

$$
h_{n} \rightarrow h, \quad g_{n} \rightarrow g, \quad g_{n}^{-1} \circ h_{n}^{-1} \circ g_{n}=g_{n}^{-1} \circ P \rightarrow g^{-1} \circ h^{-1} \circ g=g^{-1} \circ P,
$$

where

$$
\begin{aligned}
a(h) & =a, \quad r(h) & =r, & k(h)=\frac{a-\lambda r}{r-\lambda a}, \\
p & =a(g), \quad r & =r(g), & k(g)=\frac{p-\lambda r}{r-\lambda p} .
\end{aligned}
$$

Hence $g^{-1} \circ h^{-1} \circ g(r)=r$, or $h^{-1} \circ P(r)=r$. This is impossible. If $\tilde{r}=\lambda p$, since $k\left(g_{n}\right) \rightarrow+\infty$ it follows that

$$
g_{n}^{-1} \circ h_{n} \circ g_{n}\left(r_{n}\right) \rightarrow \tilde{r}=\lambda p .
$$

On the other hand,

$$
P^{-1} \circ h_{n}\left(r_{n}\right)=P^{-1}\left(r_{n}\right)=\lambda^{2} r_{n} \rightarrow \lambda^{2} r .
$$

This is a contradiction. Hence $(a, p, r) \in S_{1}$, and $S_{1}$ is both open and closed in $S$. We deduce that $S=S_{1}$. Now assume that $(a, p, r)$ and $(h, g)$ satisfy (3.1) and (3.2). By Lemma 1 we see that $h$ is determined uniquely by $a$ and $r$. The uniqueness of $g$ follows from (3.4). The other assertion of the lemma follows from Lemma 1. Q.E.D.

Similarly, we have the following assertion: 
Lemma 2'. Let $P^{\prime}(z)=\lambda^{2} z, \lambda>1, z \in \mathbf{C}$. Given three points $a, p$ and $r$ on the real axis satisfying

$$
a<p<r<\lambda^{-1} a<0,
$$

there exists a unique pair $(h, g) \in \mathscr{D}$ such that

$$
h \circ g^{-1} \circ h^{-1} \circ g=P^{\prime}, \quad \text { and } \quad a(h)=a, \quad r(h)=r, \quad a(g)=p .
$$

Conversely, if $(h, g) \in \mathscr{D}$ satisfies $h \circ g^{-1} \circ h^{-1} \circ g=P^{\prime}, r(g)<0$, then

$$
a(h)<a(g)<r(h)<\lambda^{-1} a(h) .
$$

Basing on Lemma 2 and Lemma $2^{\prime}$, we are now able to give $T_{2}$ a new parametrization. Let $p=\left(h_{i}, g_{i}\right)$ be a point in $T_{2}$. By Lemma 2 and Lemma $2^{\prime}$, we can associate with it a point $L(p)=\left(t_{1}, \ldots, t_{6}\right)$ in $\mathbf{R}_{+}^{6}$, where

$$
\begin{aligned}
t_{1}=-a\left(h_{1}\right), \quad \frac{a\left(h_{1}\right)\left(1+t_{2}\right)}{1+\lambda t_{2}} & =r\left(h_{1}\right), \quad \frac{a\left(h_{1}\right)+t_{3} r\left(h_{1}\right)}{t_{3}+1}=a\left(g_{1}\right), \\
\lambda & =\sqrt{k\left(h_{1} \circ g_{1}^{-1} \circ h_{1}^{-1} \circ g_{1}\right)}=t_{4}+1, \\
\frac{1+t_{5} \lambda}{t_{5}+1} & =r\left(h_{2}\right), \quad \frac{1+t_{6} r\left(h_{2}\right)}{t_{6}+1}=a\left(g_{2}\right) .
\end{aligned}
$$

Theorem 1. The mapping $L: T_{2} \rightarrow \mathbf{R}_{+}{ }^{6}$ is a real analytic diffeomorphism.

Proof. The mapping $L$ is obviously real analytic. To see it is injective, we only have to verify that $L(p)=\left(t_{1}, \ldots, t_{6}\right)$ determines $p$ uniquely for any $p=\left(h_{i}, g_{i}\right)$ in $T_{2}$. But this is true by Lemma 2 and Lemma $2^{\prime}$. Assume that $\left(t_{1}, \ldots, t_{6}\right)$ as above is the image of $p=\left(h_{i}, g_{i}\right) \in T_{2}$ under $L$. For any $t>0$, we want to show that $\left(t t_{1}, t_{2}, \ldots, t_{6}\right)$ is the image of a point in $T_{2}$. The set $T=\left\{t>0:\left(t t_{1}, t_{2}, \ldots, t_{6}\right) \in\right.$ $\left.T_{2}\right\}$ is open in $\mathbf{R}_{+}$by the invariance of domain. Without loss of generality, we may assume that $t>1$ and for any $1<t^{\prime}<t,\left(t^{\prime} t_{1}, t_{2}, \ldots, t_{6}\right)=L\left(p\left(t^{\prime}\right)\right)$, where $p\left(t^{\prime}\right)=\left(h_{i}\left(t^{\prime}\right), g_{i}\left(t^{\prime}\right)\right) \in T_{2}$. If $\left(t t_{1}, t_{2}, \ldots, t_{6}\right) \notin L\left(T_{2}\right)$, then $p\left(t^{\prime}\right) \rightarrow \partial T_{2}$. Since the geodesics on $R$ "covered" by $h_{1}^{0}, g_{1}^{0}, h_{2}^{0}, g_{2}^{0}$, and $h_{1}^{0} \circ h_{2}^{0-1}$ fill up the surface $R$, the sum $s\left(t^{\prime}\right)=k\left(h_{1}\left(t^{\prime}\right)\right)+k\left(g_{1}\left(t^{\prime}\right)\right)+k\left(h_{2}\left(t^{\prime}\right)\right)+k\left(g_{2}\left(t^{\prime}\right)\right)+k\left(h_{1}\left(t^{\prime}\right) \circ h_{2}^{-1}\left(t^{\prime}\right)\right)$ must approach infinity when $t^{\prime}$ tends to $t$ according to Lemma 3.1 in [4]. On the other hand, we see from (3.5), Lemma 1 and the proof in Lemma 2 that $k\left(h_{1}\left(t^{\prime}\right)\right)$, $k\left(g_{1}\left(t^{\prime}\right)\right), k\left(h_{2}\left(t^{\prime}\right)\right)$, and $k\left(g_{2}\left(t^{\prime}\right)\right)$ are all bounded. So $k\left(h_{1}\left(t^{\prime}\right) \circ h_{2}^{-1}\left(t^{\prime}\right)\right)$ must approach infinity when $t^{\prime}$ tends to $t$. Since $a\left(h_{j}\left(t^{\prime}\right)\right), r\left(h_{j}\left(t^{\prime}\right)\right)$ and $k\left(h_{j}\left(t^{\prime}\right)\right)$ are all bounded, it follows that the determinant of $\tilde{h}_{1}\left(t^{\prime}\right) \circ \tilde{h}_{2}^{-1}\left(t^{\prime}\right)$ approaches 0 when $t^{\prime}$ approaches $t$, where

$$
\begin{aligned}
\tilde{h}_{1}\left(t^{\prime}\right) & =\left(\begin{array}{cc}
a\left(h_{1}\left(t^{\prime}\right)\right) k\left(h_{1}\left(t^{\prime}\right)\right)-r\left(h_{1}\left(t^{\prime}\right)\right) & -a\left(h_{1}\left(t^{\prime}\right)\right) r\left(h_{1}\left(t^{\prime}\right)\right)\left(k\left(h_{1}\left(t^{\prime}\right)\right)-1\right) \\
k\left(h_{1}\left(t^{\prime}\right)\right)-1 & a\left(h_{1}\left(t^{\prime}\right)\right)-k\left(h_{1}\left(t^{\prime}\right)\right) r\left(h_{1}\left(t^{\prime}\right)\right)
\end{array}\right), \\
\tilde{h}_{2}^{-1}\left(t^{\prime}\right) & =\left(\begin{array}{cc}
r\left(h_{2}\left(t^{\prime}\right)\right) k\left(h_{2}\left(t^{\prime}\right)\right)-a\left(h_{2}\left(t^{\prime}\right)\right) & -r\left(h_{2}\left(t^{\prime}\right)\right) a\left(h_{2}\left(t^{\prime}\right)\right)\left(k\left(h_{2}\left(t^{\prime}\right)\right)-1\right) \\
k\left(h_{2}\left(t^{\prime}\right)\right)-1 & r\left(h_{2}\left(t^{\prime}\right)\right)-k\left(h_{2}\left(t^{\prime}\right)\right) a\left(h_{2}\left(t^{\prime}\right)\right)
\end{array}\right) .
\end{aligned}
$$


By a straightforward computation we get

$$
\begin{aligned}
\operatorname{det}\left(\tilde{h}_{1}\left(t^{\prime}\right) \tilde{h}_{2}^{-1}\left(t^{\prime}\right)\right)= & k\left(h_{1}\left(t^{\prime}\right)\right) k\left(h_{2}\left(t^{\prime}\right)\right) . \\
& \cdot\left[r\left(h_{1}\left(t^{\prime}\right)\right)-a\left(h_{1}\left(t^{\prime}\right)\right)\right]^{2}\left[r\left(h_{2}\left(t^{\prime}\right)\right)-a\left(h_{2}\left(t^{\prime}\right)\right)\right]^{2} .
\end{aligned}
$$

This is a contradiction. Keeping to the same analysis on $t_{2}, \ldots$, and $t_{6}$, we can prove that any point in $\mathbf{R}_{+}^{6}$ is the image of a point in $T_{2}$, i.e., $L$ is surjective. Q.E.D.

Corollary 1. Assume that $g, h \in \mathscr{D}$ satisfy (3.2). Then the group generated by $g$ and $h$ is discrete.

Corollary 2. Let $G$ be a group generated by four hyperbolic transformations $h_{1}, g_{1}, h_{2}$, and $g_{2}$, and assume that $\left[h_{1}, g_{1}\right]=\left[h_{2}, g_{2}\right]^{-1}$ is also hyperbolic. If the axes of $h_{j}, g_{j}$, and $\left[h_{1}, g_{1}\right]$ form a picture conjugate to Figure 1 , then $G$ is discrete.

Let $\mathbf{R}_{+}^{6}$ be our parameter space of $T_{2}$. Given a point $\left(t_{1}, \ldots, t_{6}\right)$ in $\mathbf{R}_{+}^{6}$, we can get the generators for the corresponding marked group in the following way. First of all, $h_{1}$ and $h_{2}$ can easily be defined by (3.5), Lemma 1 and (2.1). For obtaining $g_{1}$ and $g_{2}$ we need more calculations. From $h_{2} \circ g_{2}^{-1} \circ h_{2}^{-1} \circ g_{2}=P$ we have

$$
g_{2}\left(P_{a}\right)=r\left(h_{2}\right), \quad g_{2}\left(P_{r}\right)=a\left(h_{2}\right)
$$

where

$$
\begin{aligned}
& P_{a}=a\left(h_{2}^{-1} \circ P\right)=\left[2\left(1-k\left(h_{2}\right)\right)\right]^{-1}\left(a\left(h_{2}\right)-k\left(h_{2}\right) r\left(h_{2}\right)+\lambda^{2}\left(r\left(h_{2}\right)-k\left(h_{2}\right) a\left(h_{2}\right)\right)\right. \\
& \left.+\sqrt{\left(a\left(h_{2}\right)-k\left(h_{2}\right) r\left(h_{2}\right)+\lambda^{2}\left(r\left(h_{2}\right)-k\left(h_{2}\right) a\left(h_{2}\right)\right)\right)^{2}-4 \lambda\left(k\left(h_{2}\right)-1\right)^{2} a\left(h_{2}\right) r\left(h_{2}\right)}\right) \\
& P_{r}=r\left(h_{2}^{-1} \circ P\right)=\left[2\left(1-k\left(h_{2}\right)\right)\right]^{-1}\left(a\left(h_{2}\right)-k\left(h_{2}\right) r\left(h_{2}\right)+\lambda^{2}\left(r\left(h_{2}\right)-k\left(h_{2}\right) a\left(h_{2}\right)\right)\right. \\
& -\sqrt{\left.\left(a\left(h_{2}\right)-k\left(h_{2}\right) r\left(h_{2}\right)+\lambda^{2}\left(r\left(h_{2}\right)-k\left(h_{2}\right) a\left(h_{2}\right)\right)\right)^{2}-4 \lambda\left(k\left(h_{2}\right)-1\right)^{2} a\left(h_{2}\right) r\left(h_{2}\right)\right)} .
\end{aligned}
$$

Let

$$
\begin{array}{ccc}
a_{2}=a\left(g_{2}\right), & r_{2}=r\left(g_{2}\right), \quad k_{2}=k\left(g_{2}\right), \\
\tilde{a}_{2}=a\left(h_{2}\right), \quad \tilde{r}_{2}=r\left(h_{2}\right) . &
\end{array}
$$

By (3.6) and Lemma 1, we get

$$
\left\{\begin{array}{l}
\left(a_{2}-\tilde{a}_{2}\right)\left(P_{r}-\lambda a_{2}\right) k_{2}+\left(a_{2}+\lambda a_{2}-\lambda \tilde{a}_{2}-P_{r}\right) r_{2}=a_{2}^{2}-\tilde{a}_{2} P_{r} \\
\left(a_{2}-\tilde{r}_{2}\right)\left(P_{a}-\lambda a_{2}\right) k_{2}+\left(a_{2}+\lambda a_{2}-\lambda \tilde{r}_{2}-P_{a}\right) r_{2}=a_{2}^{2}-\tilde{r}_{2} P_{a} .
\end{array}\right.
$$

Solving the system of equations we get $k_{2}$ and $r_{2}$, which together with $a_{2}$ give $g_{2}$. The transformation $g_{1}$ can be obtained in a similar way. 


\section{References}

[1] Chen, M.: Decompositions of Teichmüller space by geodesic length mappings. - Ann. Acad. Sci. Fenn. Ser. A I Math. Dissertations 82, 1991, 1-31.

[2] Lento, O.: Univalent functions and Teichmüller spaces. - Springer-Verlag, New York, 1987.

[3] Li, ZHoNG: Quasiconformal mappings and their applications to the theory of Riemann surfaces. - Science Press, Beijing, 1988 (Chinese).

[4] Kerckhoff, S.P.: The Nielsen realization problem. - Ann. of Math. 117, 1983, 235-265.

[5] MASKIT, B.: Parameters for Fuchsian groups I-Signature $(0,4)$. - Holomorphic functions and moduli, II, 251-265. Math. Sci. Res. Inst. Pub. 11. Springer-Verlag, New York, 1988.

[6] Maskit, B.: Parameters for Fuchsian groups II-Topological type (1,1). - Ann. Acad. Sci. Fenn. Ser. A I Math. 14, 1989, 265-275.

[7] Sepp ̈̈L̈̈, M., and T. SoRVAli: Traces of commutators of Möbius transformations. Math. Scand. 68, 1991, 53-58.

Received 3 October 1991 